\title{
Common module analysis reveals prospective targets and mechanisms of pediatric adrenocortical adenoma and carcinoma
}

\author{
ANURAG KULSHRESTHA $^{1}$ and SHIKHA SUMAN ${ }^{2}$ \\ ${ }^{1}$ Bioinformatics Division, National Bureau of Animal Genetic Resources, Karnal, Haryana 132001;
${ }^{2}$ Department of Applied Sciences, Indian Institute of Information Technology, Allahabad, Uttar Pradesh 211012, India
}

Received May 5, 2016; Accepted June 9, 2017

DOI: $10.3892 / 01.2017 .7646$

\begin{abstract}
Pediatric adrenocortical carcinoma and adrenocortical adenoma are two rare diseases affecting children. Molecular analyses were performed to identify commonalities in gene expression between the diseases. Differentially expressed genes were identified for the pediatric adrenocortical adenoma and carcinoma tissues, as compared with normal tissues, using the expression dataset. Protein-protein interaction (PPI) networks were constructed for adenoma and carcinoma disease models, and common modules among the diseases were identified. A total of two common modules with 14 nodes and 20 nodes were revealed among the adenoma and carcinoma networks, respectively. Genes of the common modules were also identified to be the common hub genes of the disease models. Enrichment of the genes of the common modules suggested associations with steroid biosynthesis, the proteasome, cell cycle and metabolic pathways. Modularity, topological and functional analysis of the PPI networks revealed common modules among pediatric adenoma and carcinoma disease models, which provided insight into the underlying disease mechanisms and suggesting prospective targets for future study.
\end{abstract}

\section{Introduction}

Pediatric adrenocortical tumors (ACT) are a rare but aggressive endocrine malignancy accountable for $\sim 0.2 \%$ of childhood cancer cases (1). ACTs comprise benign adenomas and highly malignant carcinomas whose pathogenesis remains incompletely understood (2). The majority of pediatric ACTs are functional, with symptoms of excessive androgen production, in comparison to adult ACT (1). Though histopathological demarcation between adenoma and carcinoma is

Correspondence to: Miss Shikha Suman, Department of Applied Sciences, Indian Institute of Information Technology, IIIT Road, Devghat, Jhalwa, Allahabad, Uttar Pradesh 211012, India

E-mail: shikha.suman27@gmail.com

Key words: pediatric adrenocortical carcinoma, pediatric adrenocortical adenoma, hubs, modules complicated, $80-90 \%$ of pediatric ACTs are carcinomas (1). Complete surgical resection remains the only potent treatment for pediatric ACT (3).

The elements responsible for sporadic pediatric ACT remain unknown, yet the resemblance of cases of ACT to cases with inherent susceptibility indicates a common method of tumorigenesis (4). Germline TP53 mutations (Li-Fraumeni syndrome) or genetic and/or epigenetic modifications affecting chromosome 11p15 (Beckwith-Wiedemann syndrome) are commonly associated with childhood ACT (4). Insulin-like growth factor 2 (IGF2) overexpression has been identified in pediatric adrenocortical adenoma and carcinoma. In addition, IGF1R mRNA levels have been demonstrated to be higher in pediatric adrenocortical carcinomas (ACC) (5). Carcinomas have been revealed to possess more chromosomal alterations when compared with adenomas (6). In a prior study, it was hypothesized that a number of genomic changes are responsible for progression from normal tissue, to adenoma to carcinoma (7).

A considerable number of disease-associated genes have been identified by expression studies in previous years (8). In the present study, bioinformatics methodologies, such as protein-protein interaction (PPI) network analyses (9), that are based upon gene-encoded proteins interactions and gene module analysis have been utilized to investigate the underlying biological processes of progressing pediatric adrenocortical adenoma. In the present study, differentially expressed genes (DEGs) from pediatric adrenocortical adenoma and carcinoma were utilized to construct PPI networks based on gene-encoded protein interaction information. The networks were analyzed to identify common nodes with high connectivity (hubs) among the adenoma and carcinoma PPI networks, in order to isolate the genes responsible for disease progression. Also, an overlapping gene module among adenoma and carcinoma was identified to obtain prospective molecules associated with the progressing disease. Additionally, the association between common disease modules and pediatric ACC was outlined.

\section{Materials and methods}

Gene expression data. The raw gene expression data was retrieved from Gene Expression Omnibus (GEO; www.ncbi.nlm.nih.gov/geo/). The chip dataset GSE75415 (10) included 7 samples from healthy individuals, 5 samples 
from pediatric adrenocortical adenoma and 18 samples from pediatric ACC. Gene expression profiling for adenoma and carcinoma were performed using Affymetrix Human Genome U133 array (HG-U133A) chips (Affymetrix; Thermo Fisher Scientific, Inc., Waltham, MA, USA).

Screening the DEGs. Pre-processing and normalization were performed to remove noise from the expression dataset. For the normalization and summarization of the expression dataset, Robust Multiarray Averaging (11) in Affy package version 1.46.1 (Bioconductor, Buffalo, NY, USA) (12) of R was utilized. Linear Models for Microarray data (Limma) version 3.24.15 (Bioconductor) (13) package of $\mathrm{R}$ was employed to explore the normalized data for differential analysis. In order to obtain the adjusted P-values, multiple hypothesis testing correction was performed using the Benjamini-Hochberg (14) method. Fold-change $>2$ and adjusted $\mathrm{P}<0.05$ were considered the be demarcating parameters for the identification of DEGs in adenoma and carcinoma, as compared with in normal tissues.

Construction of PPInetworks. The extracted DEGs of adenoma and carcinoma were mapped to construct PPI networks. The Search Tool for Retrieval of Interacting Genes/Proteins (15) v10 database (https://string-db.org/) was explored to identify the interacting proteins and elucidate their function at a molecular level. Interacting proteins with a confidence score $>0.4$ were selected for constructing PPI networks and were visualized in Cytoscape version 3.2.0 (National Institute of General Medical Sciences, Bethesda, MD, USA) (16). Network topological parameters, such as network density, network centralization and network diameter, were evaluated with the Network Analyzer (17) plug-in of Cytoscape. Common hub genes among the PPI networks were also identified as being those with a connectivity degree $>10$. For the construction of the common sub-network, overlapping nodes and edges were mined by comparing the nodes and edges of the adenoma and carcinoma disease networks.

Identification of common modules. The common sub-network among adenoma and carcinoma was scrutinized to explore high modularity clusters in the network, as genes in the module tend to possess similar biological function. The Molecular Complex Detection (MCODE) (18) plug-in of Cytoscape was employed to identify the functional modules in the disease system with degree cutoff $=2, \mathrm{k}$ - score $=2$, maximum depth $=100$ and node score cutoff $=0.2$. Modules with an MCODE score $\geq 4$ and nodes $\geq 6$ were considered for additional analysis. Furthermore, overlapping genes/proteins shared between common modules and common hubs of the adenoma and carcinoma PPI networks were also identified.

Enrichment analysis. Kyoto Encyclopedia of Genes and Genomes (KEGG) pathway and Gene Ontology (GO) enrichment analysis was performed through WEB-based gene set analysis toolkit (WebGestalt; http://www.webgestalt.org/) (19) on genes in the common module, to identify the biological processes and pathways underlying the disease system. WebGestalt incorporates proteomic, genomic and functional categories from numerous public resources. The
Benjamini-Hochberg method was utilized to obtain adjusted $\mathrm{P}$-values. The cut-off criteria included $\mathrm{P}<0.05$ and number of genes $>2$.

\section{Results}

Identification of DEGs. Identifying and analyzing the dysregulated genes was indispensable for evaluation of the common underlying mechanisms of ACT adenoma and carcinoma. A total of 550 DEGs, including 376 upregulated and 174 downregulated genes, were identified in adrenocortical adenoma tissues compared with in healthy individuals, with an adjusted $\mathrm{P}<0.05$ and fold-change $>2$. Additionally, $431 \mathrm{DEGs,}$ including 228 upregulated and 203 downregulated genes, were documented for ACC, as compared with in normal tissues, using the aforementioned predefined thresholds. A total of 228 common DEGs were also identified among pediatric adrenocortical adenoma and carcinoma (Fig. 1).

PPI network construction. The PPI network for adenoma was formed with 464 nodes and 1,947 edges with a confidence score $>0.4$. In addition, a PPI network with 366 nodes and 1,858 edges was constructed for carcinoma. The PPI networks followed a power law of distribution with $\mathrm{R}^{2}=0.841$ and 0.836 for adenoma and carcinoma, respectively. Various network parameters for the adenoma and carcinoma PPI networks are summarized in Table I. A total of 53 common hub genes were identified among adenoma and carcinoma PPI networks, with connectivity degrees $>10$ (Table II). A common sub-network with 186 nodes and 544 edges was identified by scrutinizing overlapping nodes and edges among the two PPI networks (Fig. 2).

Common module identification. As an important step for identifying the common functional module among adenoma and carcinoma disease models, the common sub-network among adenoma and carcinoma was additionally explored. A total of two common functional modules, module-1 (MCODE score $=12$ ) with 14 nodes and module-2 (MCODE score $=6.421$ ) with 20 nodes, were observed among the PPI networks of adenoma and carcinoma (Fig. 2). Notably, 12 genes of module-1 and 13 genes of module- 2 were upregulated in adenoma and in carcinoma. Additionally, the majority of the genes of the common modules were included in the common hub genes of PPI networks (Table II).

Functional enrichment of modules. Utilizing the recommended threshold of $\mathrm{P}<0.05$ and number of genes $>2$, the KEGG pathway enrichment analysis revealed the association of the genes in module-1 with steroid biosynthesis and metabolic pathways. Genes in module-2 were enriched in proteasome and cell cycle pathways (Table III). GO enrichment analysis identified major biological processes, cellular components and molecular functions associated with the genes of module-1 and module-2 (Fig. 3).

\section{Discussion}

The present study aimed to recognize the genes that serve an important role in the advancement of disease from normal, to 
Table I. Topological parameters of adenoma and carcinoma PPI networks.

\begin{tabular}{lcccccccc}
\hline PPI network & $\begin{array}{c}\text { No. of } \\
\text { nodes }\end{array}$ & $\begin{array}{c}\text { No. of } \\
\text { edges }\end{array}$ & $\mathrm{R}^{2}$ & Correlation & $\begin{array}{c}\text { Clustering } \\
\text { coefficient }\end{array}$ & $\begin{array}{c}\text { Network } \\
\text { centralization }\end{array}$ & $\begin{array}{c}\text { Network } \\
\text { density }\end{array}$ & $\begin{array}{c}\text { Network } \\
\text { diameter }\end{array}$ \\
\hline Adenoma & 467 & 1947 & 0.841 & 0.800 & 0.254 & 0.177 & 0.018 \\
Carcinoma & 366 & 1858 & 0.836 & 0.880 & 0.304 & 0.214 & 0.028 \\
\hline
\end{tabular}

PPI, protein-protein interaction.

Table II. Common hubs and common module genes among adenoma and carcinoma protein-protein interaction networks

\begin{tabular}{ll}
\hline Type Gene symbol & Genol
\end{tabular}

Common hub genes

GAPDH, ACLY, AURKA, FOS, HDAC1, EGR1, CAT, MMP2, SIRT1, SREBF1, H2AFZ, SKP2, CDC23, SCD, HMGCR, BUB3, TCP1, TXN, JUNB, CREM, FDFT1, DICER1, PSMD14, FDPS, PSMB2, SQLE, HMGCS1, DHCR7, DCN, CYP51A1, IGF2, PSMB4, PSMB7, SPARC, NR4A1, MSMO1, HSD3B2, ACAT2, MARS, LSS, SC5DL, TARS, PGD, RAD23B, VCAN, NR4A2, GEM, CANX, PTGIS, MAPK6, FOSB, IARS, CDH2

Common module genes SQLE, PTGIS, SCD, HSD3B2, DHCR7, MSMO1, C14orf1, HMGCR, FDFT1, SC5DL, LSS, CYP51A1, HMGCS1, FDPS, ACLY, SKP2, PSMD14, GAPDH, NR4A1, EGR1, JUNB, PSMB2, CREM, PSMB7, VCAN, PSMB4, DCN, CDC23, BUB3, SPARC, LUM, ODC1, GMNN, AURKA

Table III. Top five enriched KEGG pathways of the genes of the common modules.

\begin{tabular}{|c|c|c|c|c|}
\hline Term & Category & Description & Gene count & Adjusted P-value \\
\hline \multicolumn{5}{|l|}{ Module 1} \\
\hline KEGG & hsa00100 & Steroid biosynthesis & 7 & $9.42 \times 10^{-21}$ \\
\hline KEGG & hsa01100 & Metabolic pathways & 12 & $1.29 \times 10^{-17}$ \\
\hline KEGG & hsa00900 & Terpenoid backbone biosynthesis & 3 & $1.24 \times 10^{-8}$ \\
\hline \multicolumn{5}{|l|}{ Module 2} \\
\hline KEGG & hsa03050 & Proteasome & 4 & $2.26 \times 10^{-8}$ \\
\hline KEGG & hsa04110 & Cell cycle & 3 & $6.38 \times 10^{-5}$ \\
\hline KEGG & hsa04114 & Oocyte meiosis & 2 & $2.00 \times 10^{-3}$ \\
\hline KEGG & hsa04120 & Ubiquitin mediated proteolysis & 2 & $2.30 \times 10^{-3}$ \\
\hline KEGG & hsa01100 & Metabolic pathways & 3 & $1.47 \times 10^{-2}$ \\
\hline
\end{tabular}

KEGG, Kyoto Encyclopedia of Genes and Genomes; hsa, homo sapiens.

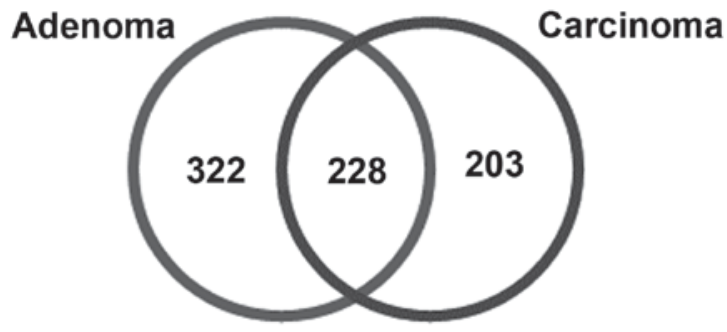

Figure 1. Common DEGs among pediatric adrenocortical adenoma and carcinoma. Values correspond to the number of DEGs in each disease, and those that are common to both $(n=228)$. DEGs, differentially expressed genes.

adenoma, to carcinoma. This required an integrative approach combining differential expression and interaction network analyses. In the present study, two networks were constructed based on the differential expression of gene-encoded protein interaction information. The degree (number of connected nodes to the disease specific nodes) of disease-associated genes is appreciably higher compared with the degree of the overall human interactome, proposing their significant role when compared with other genes. The data also support the hypothesis that disease-associated genes possess higher numbers of connections $(20,21)$. A total of 53 genes were identified to be common hubs of the disease system, and may perform important biological functions.

A total of 228 common DEGs were identified among pediatric adrenocortical adenoma and carcinoma. There were two common functional modules identified within the adenoma and carcinoma disease models. Pathway enrichment of the 


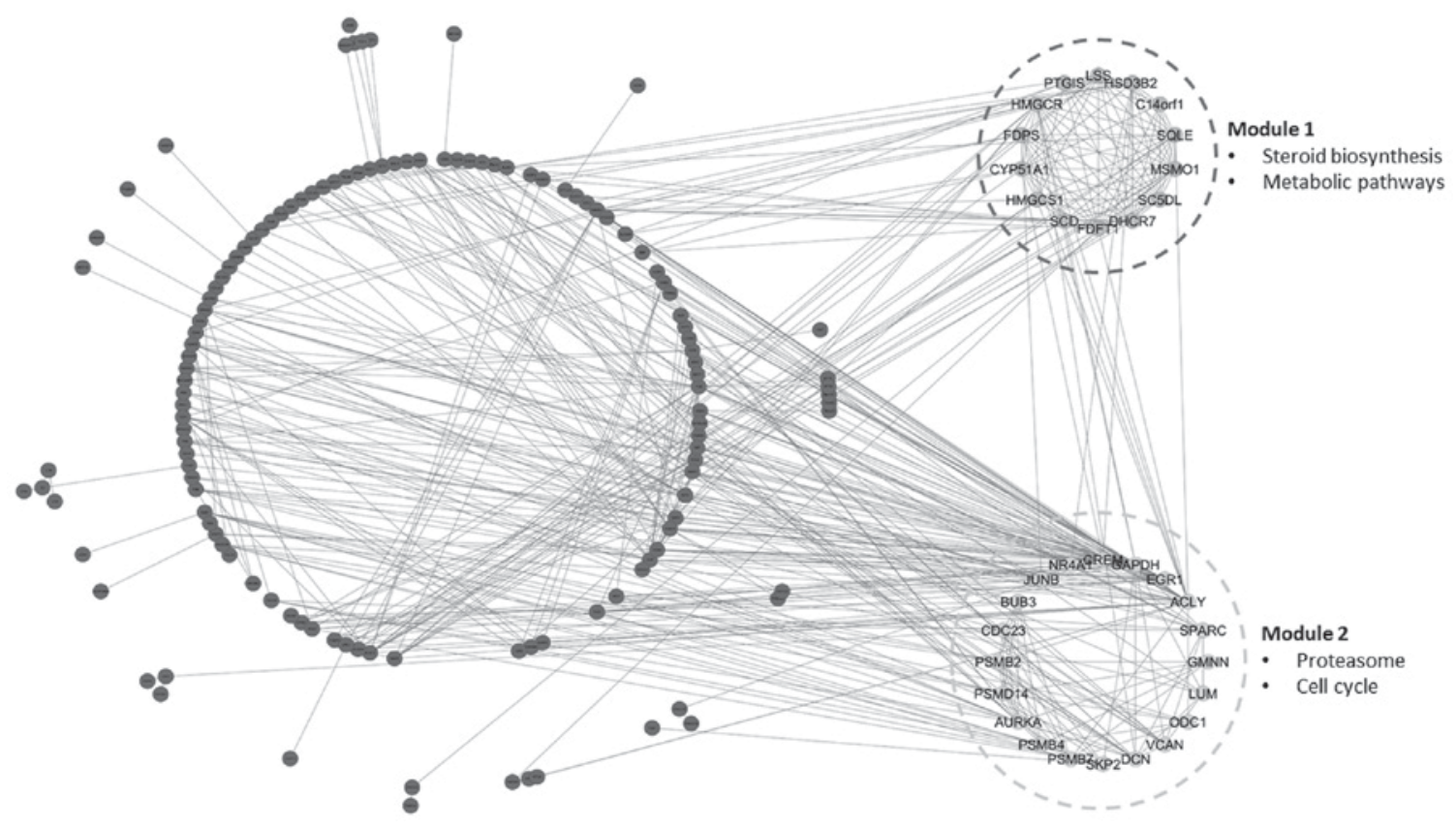

Figure 2. Common sub-network and modules identified within the adenoma and carcinoma disease models.

\section{Biological process}

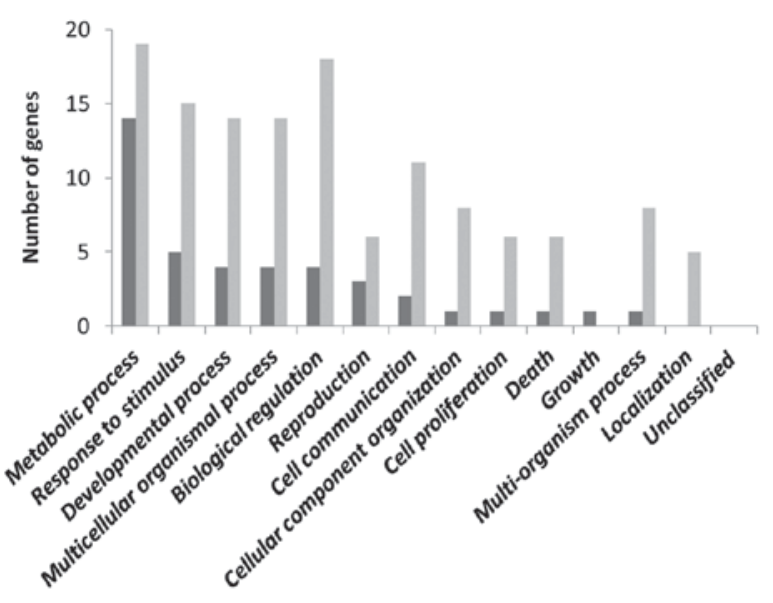

\section{Molecular function}

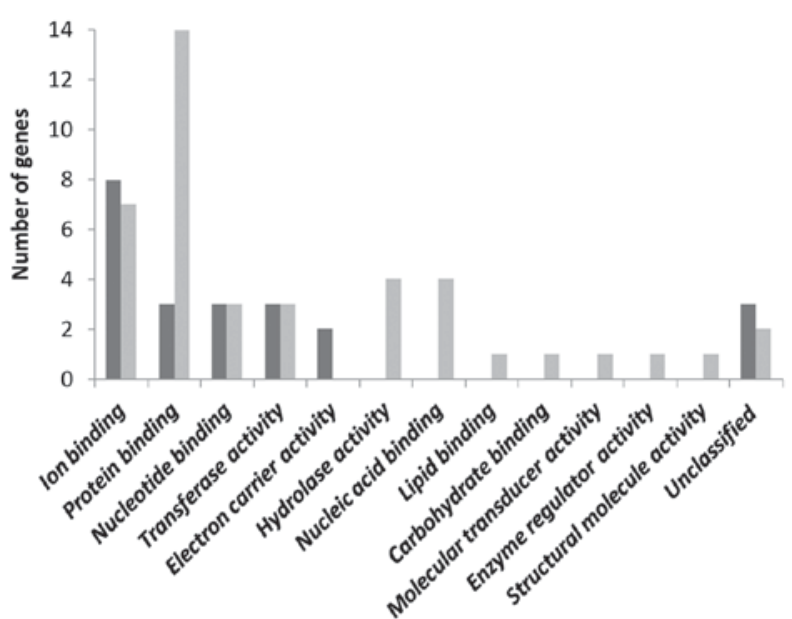

\section{Cellular component}

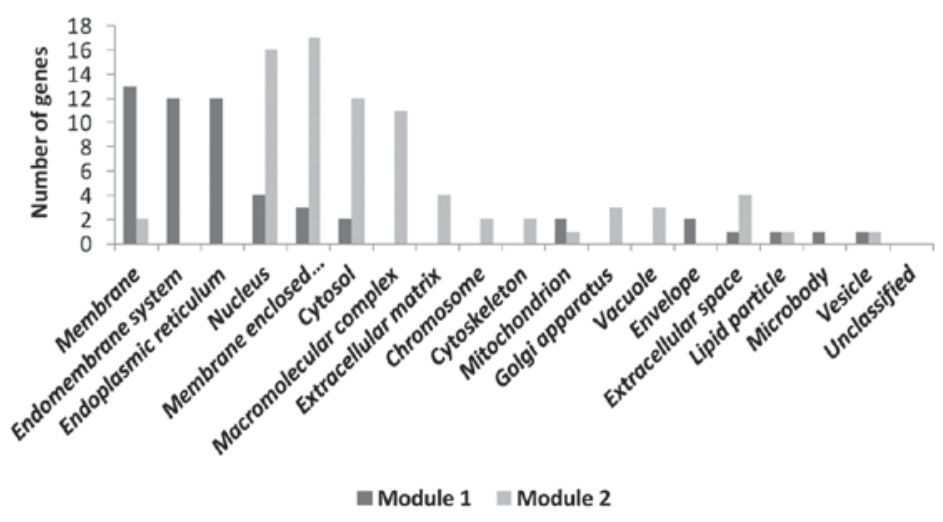

Figure 3. Enriched gene ontology categories of the genes in the common modules.

genes in the common module-1 revealed that the genes were enriched in steroid biosynthesis and metabolic pathways.
Genes in common module- 2 were associated with the proteasome and cell cycle-associated pathways. The result indicates 
that there are two common gene modules that support steroid biosynthesis, proteasome, metabolic pathways and cell cycle, which are associated with adenoma and carcinoma. These common modules may provide prospective molecular bases of disease progression. Nodes of the common modules were identified to possess greater average degrees compared with other nodes of the disease models, indicating that the genes in the common modules may be markedly more associated with disease processes compared with other genes in the disease system. Overlap between common module genes and common hubs of networks suggest that the deregulation of steroid biosynthesis, metabolic pathways, proteasome and cell cycle may assist in the development and progression of this disease. These pathways also present prospective targets for future research.

In the present study, steroid biosynthesis was one of the affected pathways. Virilization and androgen hypersecretion, primarily of cortisol, due to hormone excesses may be observed in $\sim 90 \%$ of pediatric patients, providing an improved prognosis compared with adult patients (22). It has been proposed that targeting steroidogenic factor-1 may decrease cortisol production and provide an antitumor effect (23). Measurements of dehydroepiandrosterone sulfate, hydroxyprogesterone, aldosterone, urinary 17-ketosteroids and 17-hydroxycorticosteroid, testosterone, plasma cortisol and free cortisol are routinely evaluated for suspected patients with ACT (24). In the present study, proteasome-associated pathways were also demonstrated to be affected by this disease model. The $\mathrm{Wnt} / \beta$-catenin signaling pathway is crucial for cellular growth and regulation of the adrenal gland (25). Abnormal accumulation of $\beta$-catenin (CTNNB1) causes activation of the Wnt signaling pathway and prevents its degradation by the ubiquitin-proteasome system (25). CTNNB1-activating mutations have been commonly identified in pediatric ACTs (4). Additionally, it has been proposed that Wnt signaling inhibitors may assist in the treatment of childhood ACC (26). Cell cycle-associated pathways were revealed to be commonly affected by the disease system in the present study. TP53 is a tumor suppressor gene involved in cell cycle regulation, thereby causing cell cycle arrest or cell death by DNA damage. Germline TP53 mutation is markedly associated with pediatric ACT by promoting chromosomal instability (4). It has been proposed that loss of the normal inhibitory role of TP53 protein in the cell cycle is associated with the development of ACC (27).

The majority of the genes of common module-1 and module- 2 are located in the membrane and membrane-enclosed lumen, respectively, suggesting prospective targets for disease treatment. Genes of module- 1 and module- 2 are enriched in biological processes associated with metabolism, which provides mechanisms for additional studies. The enriched molecular functions, such as ion binding and protein binding, represent prospective drug targets.

In conclusion, pediatric adrenocortical adenoma and carcinoma disease models were explored to identify common hub genes among adenoma and carcinoma, which serve roles in disease-associated pathological processes. Additionally, two common gene modules were identified among the adenoma and carcinoma networks. The genes of these modules were also the common hubs of the disease model. The present study exhibited certain limitations, as the entire study was based on a bioinformatics approache, and the results must be verified by further experiments.

\section{References}

1. Gulack BC, Rialon KL, Englum BR, Kim J, Talbot LJ, Adibe OO, Rice HE and Tracy ET: Factors associated with survival in pediatric adrenocortical carcinoma: An analysis of the National Cancer Data Base (NCDB). J Pediatr Surg 51: 172-177, 2016.

2. Ronchi CL, Sbiera S, Leich E, Henzel K, Rosenwald A, Allolio B and Fassnacht M: Single nucleotide polymorphism array profiling of adrenocortical tumors-evidence for an adenoma carcinoma sequence? PLoS One 8: e73959, 2013.

3. Pinto EM, Morton C, Rodriguez-Galindo C, McGregor L, Davidoff AM, Mercer K, Debelenko LV, Billups C, Ribeiro RC and Zambetti GP: Establishment and characterization of the first pediatric adrenocortical carcinoma xenograft model identifies topotecan as a potential chemotherapeutic agent. Clin Cancer Res 19: 1740-1747, 2013.

4. Pinto EM, Chen X, Easton J, Finkelstein D, Liu Z, Pounds S, Rodriguez-Galindo C, Lund TC, Mardis ER, Wilson RK, et al: Genomic landscape of paediatric adrenocortical tumours. Nat Commun 6: 6302, 2015.

5. Lima Lde O, Lerario AM, Alencar GA, Brito LP, Almeida MQ, Domenice S, Latronico AC, Mendonca BB and Fragoso MC: Clinical and molecular aspects of a pediatric metachronous adrenocortical tumor. Arq Bras Endocrinol Metabol 55: 72-77, 2011.

6. Lalli E and Figueiredo BC: Pediatric adrenocortical tumors: What they can tell us on adrenal development and comparison with adult adrenal tumors. Front Endocrinol (Lausanne) 6: 23, 2015.

7. Gara SK, Wang Y, Patel D, Liu-Chittenden Y, Jain M, Boufraqech M, Zhang L, Meltzer PS and Kebebew E: Integrated genome-wide analysis of genomic changes and gene regulation in human adrenocortical tissue samples. Nucleic Acids Res 43: 9327-9339, 2015.

8. Guo L, Du Y and Wang J: Network analysis reveals a stress-affected common gene module among seven stress-related diseases/systems which provides potential targets for mechanism research. Sci Rep 5: 12939, 2015.

9. Nair J, Ghatge M, Kakkar VV and Shanker J: Network analysis of inflammatory genes and their transcriptional regulators in coronary artery disease. PLoS One 9: e94328, 2014.

10. West AN, Neale GA, Pounds S, Figueredo BC, Rodriguez Galindo C, Pianovski MA, Oliveira Filho AG, Malkin D, Lalli E, Ribeiro R and Zambetti GP: Gene expression profiling of childhood adrenocortical tumors. Cancer Res 67: 600-608, 2007.

11. Irizarry RA, Hobbs B, Collin F, Beazer-Barclay YD, Antonellis KJ, Scherf U and Speed TP: Exploration, normalization and summaries of high density oligonucleotide array probe level data. Biostatistics 4: 249-264, 2003.

12. Gautier L, Cope L, Bolstad BM and Irizarry RA: affy--analysis of Affymetrix GeneChip data at the probe level. Bioinformatics 20: 307-315, 2004.

13. Smyth GK: Limma: Linear models for microarray data. In: Bioinformatics and computational biology solutions using $\mathrm{R}$ and bioconductor. Springer, New York, pp397-420, 2005.

14. Benjamini Y and Hochberg Y: Controlling the false discovery rate: A practical and powerful approach to multiple testing. J Roy Stat Soc B Met 57: 289-300, 1995.

15. Szklarczyk D, Franceschini A, Wyder S, Forslund K, Heller D, Huerta-Cepas J, Simonovic M, Roth A, Santos A, Tsafou KP, et al: STRING v10: Protein-protein interaction networks, integrated over the tree of life. Nucleic Acids Res 43 (Database Issue): D447-D452, 2015.

16. Smoot ME, Ono K, Ruscheinski J, Wang PL and Ideker T: Cytoscape 2.8: New features for data integration and network visualization. Bioinformatics 27: 431-432, 2011.

17. Assenov Y, Ramírez F, Schelhorn SE, Lengauer T and Albrecht M: Computing topological parameters of biological networks. Bioinformatics 24: 282-284, 2008.

18. Bader GD and Hogue CW: An automated method for finding molecular complexes in large protein interaction networks. BMC Bioinformatics 4: 2, 2003

19. Wang J, Duncan D, Shi Z and Zhang B: WEB-based GEne SeT AnaLysis Toolkit (WebGestalt): Update 2013. Nucleic Acids Res 41 (Web Server Issue): W77-W83, 2013. 
20. Jonsson PF and Bates PA: Global topological features of cancer proteins in the human interactome. Bioinformatics 22: 2291-2297, 2006.

21. Sun J, Jia P, Fanous AH, van den Oord E, Chen X, Riley BP Amdur RL, Kendler KS and Zhao Z: Schizophrenia gene networks and pathways and their applications for novel candidate gene selection. PLoS One 5: e11351, 2010.

22. Maluf DF, de Oliveira BH and Lalli E: Therapy of adrenocortical cancer: Present and future. Am J Cancer Res 1: 222-232, 2011.

23. Aufforth RD and Nilubol N: Emerging therapy for adrenocortical carcinoma. Int J Endocr Oncol 1: 173-182, 2014.

24. Ribeiro RC, Michalkiewicz EL, Figueiredo BC, DeLacerda L, Sandrini F, Pianovsky MD, Sampaio G and Sandrini R: Adrenocortical tumors in children. Braz J Med Biol Res 33: 1225-1234, 2000.
25. Berthon A, Martinez A, Bertherat J and Val P: Wnt/ $\beta$-catenin signalling in adrenal physiology and tumour development. Mol Cell Endocrinol 351: 87-95, 2012.

26. Gundgurthi A, Kharb S, Dutta MK, Garg MK, Khare A, Jacob MJ and Bhardwaj R: Childhood adrenocortical carcinoma: Case report and review. Indian J Endocrinol Metab 16: 431-435, 2012.

27. Pinto EM, Ribeiro RC, Figueiredo BC and Zambetti GP. TP53-associated pediatric malignancies. Genes Cancer 2: 485-490, 2011. 